adverse effect of occupational exposure. Research have suggested carcinogenic potential of some forms of CNTs (MWCNT-7 Mitsui) and asbestos-like pathogenesis. Studying epigenetic alterations (e.g. DNA methylation) could provide important additional evidence to determine CNT toxicity and disease progression.

Methods To understand epigenetic effects of CNT (SWCNT and MWCNT), we designed a translational study incorporating in vitro and in vivo experiments. The changes were compared to results of asbestos exposure study. Changes in DNA methylation were studied at global (LC/MS-MS), genome wide (illumina $450 \mathrm{~K}$ ), sequence specific levels (bisulfite pyrosequenceing). Changes in gene expression were studied using RNASeq. Finally, signatures obtained from these studies were validated in 23 workers exposed to MWCNT.

Result In vitro, CNTs and asbestos induced gene specific DNA methylation changes. Asbestos exposure induced alterations in genes associated with Rho mediated signal transduction, HOX genes, WNT genes. Methylation and transcriptomic profiles of CNT exposed cells revealed alterations in DNA damage repair, tp53, cell cycle, protein phosphorylation pathways. Additionally, CNTs induced sequence specific changes in promoter region of several key genes including DNMT1, HDAC4, ATM, MAP3K10, PIK3R2 and MYO1C. Some of the genes, specifically ATM was also differentially methylated by SWCNTs and MWCNTs in the in vivo study. Based on these result, we studied some of these markers in MWCNT exposed workers, where we observed significant changes in sequence specific methylation for DNMT1, ATM, SKI and HDAC4 promoter CpGs.

Conclusion Epigenetic cell responses provides important insights in potential health risks and underlying mechanisms. Hence, many of these genes have been associated with occupational asbestos and smoking induced diseases and cancer. Further research needs to confirm whether methylation alterations in this set of genes can be used in monitoring changes associated CNT exposure and effect.

\section{FACTORS ASSOCIATED TO RETURN TO WORK AFTER SICKNESS ABSENCE DUE TO MENTAL DISORDERS AMONG BRAZILIAN WORKERS}

${ }^{1}$ IS Silva-Junior*, ${ }^{2} \mathrm{RH}$ Griep, ${ }^{3} \mathrm{MC}$ Martinez, ${ }^{4} \mathrm{FM}$ Fischer. ${ }^{1}$ National Social Security Institute, São Paulo, Brazil; ${ }^{2}$ Oswaldo Cruz Foundation, Rio de Janeiro, Brazil; ${ }^{3}$ WAF Informática, São Paulo, Brazil; ${ }^{4}$ School of Public Health, University of São Paulo, São Paulo, Brazil

\subsection{6/oemed-2018-ICOHabstracts.415}

Introduction Mental disorders are the third leading cause for grant sickness social security benefit in Brazil. Those workers have longer time to return to work (RTW) comparing to other disabilities. This study aims to analyse factors associated to RTW after an episode of sickness absence due to mental disorders (MD).

Methods A longitudinal study was conducted in the city of São Paulo, Brazil, from 2014-2016. Included 204 workers requiring sickness social security benefit due to MD. At baseline, participants fill questionnaires about sociodemographic, health risk behaviours, work characteristics, health conditions and social security history. They were followed for 365 days after the first day of sickness absence. Survival analysis (Kaplan-Meier curve and Cox regression) were performed to identify factors influencing the first RTW.
Result The group was composed mostly by women (71.1\%), people aged under $40(67.6 \%)$ education equal or higher 12 years (80.4\%) and diagnosed as depressed (52.9\%). Many worked in customer service jobs (44.1\%), reported effortreward imbalance at work (57.4\%) and high overcommitment (74.2\%). The average time for return to work was approximately six months among the $63.0 \%$ who tried to resume their work activities. Factors associated to return to work within one year after sick leave were: aged between 30-39 years (HR 1.76; 95\% CI: 1.08 to 2.79 ), 12 or more years of education (HR 1.87; 95\% CI: 1.10 to 3.17), low alcohol intake (HR 2.65; 95\% CI: 1.75 to 4.02) and low level of anxiety symptoms at baseline (HR 0.17 ; 95\% CI: 0.04 to 0.74 ) - when analysis were adjusted by sex and job title.

Discussion Sociodemographic characteristics, risk health behaviours and medical conditions at baseline were associated to RTW after sick leave due to MD. Further studies, with larger sample, are needed to improve estimates and discussion focused in interventions to early RTW in public and private sectors. Acknowledgments: CNPq grant nº442051/2014-0.

\section{RELATIONSHIP BETWEEN SHIFT WORK AND THE ONSET OF RHEUMATOID ARTHRITIS; RESULTS FROM THE SWEDISH EIRA CASE-CONTROL STUDY}

${ }^{1,2}$ Alfredsson Lars*, ${ }^{1}$ AK Hedström, ${ }^{3} \mathrm{~T}$ Åkerstedt, ${ }^{4} \mathrm{~L}$ Klareskog. ${ }^{1}$ Institute of Environmental Medicine, Karolinska Institutet, Stockholm, Sweden; ${ }^{2}$ Centre for Occupational and Environmental Medicine, Stockholm County Council, Stockholm, Sweden; ${ }^{3}$ Stockholm University and Department of clinical neuroscience, Karolinska institutet, Stockholm, Sweden; ${ }^{4}$ Department of Medicine, Rheumatology Unit, Karolinska University Hospital, Solna, Stockholm, Sweden

\subsection{6/oemed-2018-ICOHabstracts.416}

Introduction Shift work has previously been associated with increased RA risk in females. The aim of this study was to investigate the potential association between permanent night shift work, rotating shift work, and day oriented shift work, and risk of developing anti- citrullinated peptide antibodies (ACPA) positive and ACPA negative RA.

Methods The present report is based on a Swedish population-based, case-control study with incident cases of RA (1951 cases, 2225 matched controls). Using logistic regression, occurrence of RA among subjects who have been exposed to different kinds of shift work was compared with that among those who have never been exposed, by calculating the odds ratio (OR) with a 95\% confidence interval (CI).

Result Rotating shift work and day oriented shift work were associated with a $30 \%$ increased risk of developing ACPA positive RA, but not ACPA negative RA. There was an inverse association between permanent night shift work and risk of both ACPA positive RA (OR 0.7, 95\% CI: 0.6 to 0.9 ) and APCA negative RA (OR 0.8, 95\% CI: 0.6 to 1.0 ). For both subsets of RA, significant trends showed a lower risk of developing RA with increasing duration of permanent night shift work.

Discussion Sleep restriction as a consequence of shift work is associated with several biological effects among which changes in melatonin production may be involved. The present epidemiological findings of a complex relationship between sleep patterns and different forms of RA may be of importance for increasing the understanding of the pathophysiology of RA. 


\section{USING BURDEN OF CANCER TO PROMOTE POLICY CHANGE}

\begin{abstract}
1,2PA Demers* ${ }^{1,3}$ J Kim, ${ }^{1} \mathrm{M}$ Pahwa, ${ }^{4} \mathrm{CE}$ Peters, ${ }^{1} \mathrm{C}$ Song, ${ }^{1,2} \mathrm{VH}$ Arrandale, ${ }^{5} \mathrm{HW}$ Davies, ${ }^{6} \mathrm{~F}$ Labrèche, ${ }^{6,7} \mathrm{~J}$ Lavoue, ${ }^{8} \mathrm{AM}$ Nicol, ${ }^{5} \mathrm{CB}$ McLeod. ${ }^{1}$ Occupational Cancer Research Centre, Cancer Care Ontario, Toronto, Canada; ${ }^{2}$ Dalla Lana School of Public Health, University of Toronto, Toronto, Canada; ${ }^{3}$ Department of Epidemiology, Biostatistics, and Occupational Health, McGill University, Montreal, Canada; ${ }^{4}$ Department of Health Sciences, Carleton University, Ottawa, Canada; ${ }^{5}$ School of Population and Public Health, University of British Columbia, Vancouver, Canada; ${ }^{6}$ School of Public Health, Université de Montréal, Montreal, Quebec, Canada; ' Centre de recherche du Centre hospitalier de I'Université de Montréal, Montreal, Quebec, Canada; ${ }^{8}$ Faculty of Health Sciences, Simon Fraser University, Burnaby, Canada
\end{abstract}

\subsection{6/oemed-2018-ICOHabstracts.417}

Introduction Occupational cancer is poorly recognised due to its long latency among other factors. Burden of occupational cancer projects are an effective tool for drawing attention to the magnitude of the problem and promoting prevention. We present here some major results from the Canadian Burden of Occupational Cancer project and discuss its use for prevention.

Methods Our methods are based on a similar UK project, with several enhancements. The historic labour force was identified using 1961 through 2001 Census data. Occupational exposure prevalence was estimated using CAREX Canada data, allowing calculation of levels of exposure for 44 carcinogens. Relative risks were based on large epidemiologic studies and meta-analyses representative of Canadian exposure circumstances. Standard methods with Levin's equation were used to calculate attributable fractions.

Result Exposure to solar radiation was responsible for the largest number of cancers (4550 basal and squamous cell carcinomas annually, $6.3 \%$ of all non-melanoma skin cancer). Asbestos came second with 425 mesotheliomas $(80.5 \%$ of mesothelioma, including para-occupational, but not environmental, exposure), 1900 lung cancers (8.0\% of lung cancer), and smaller numbers of larynx and, ovarian, and suspected other cancers. Diesel engine exhaust was responsible for 560 lung cancers $(2.4 \%$ of lung cancer) and suspected for 200 bladder cancers $(2.7 \%$ of bladder cancer). Crystalline silica and welding fumes were responsible for 570 and 315 lung cancers $(2.4 \%$ and $1.3 \%$ of all), respectively. Shiftwork resulting in circadian disruption was suspected for between 460 and 1180 breast cancers $(2.0 \%-5.2 \%$ of all), annually.

Discussion Results from the project have begun to be used by Canadian regulatory organisations to establish priorities and by advocacy organisations to raise awareness and push for policy change. These efforts and the input from burden estimates have played a role in the governmental campaign to ban asbestos, now scheduled for 2018.

\section{OCCUPATIONAL HEALTH PROGRAM FOR MIGRANT WORKERS IN THAILAND}

'S Arphorn*, ${ }^{2} \mathrm{P}$ Khongnil, ${ }^{3} \mathrm{G}$ Bangkadanara, ${ }^{1} \mathrm{~T}$ Thanachoksawang, ${ }^{4} \mathrm{~B}$ Wangkahad. ${ }^{1}$ Department of Occupational Health and Safety, Mahidol University, Bangkok, Thailand; ${ }^{2}$ Kanchanaburi College of Agriculture and Technology, Kanchanaburi, Thailand; ${ }^{3}$ School of Health Science, Sukhothai thammathirat Open University, Nonthaburi, Thailand; ${ }^{4}$ Syngenta Crop company, Bangkok, Thailand

10.1136/oemed-2018-ICOHabstracts.418

Introduction Migrant workers play an important role in generating productivity of Thailand presently. The employment of these migrant workers generates social and cultural interaction. Working in agriculture is common among migrant workers especially in border areas. Thailand has an occupational health working mechanism to promote and encourage work safety especially for workers in the system. Examples of this mechanism include the work of safety officers in work safety education, improving working environment, identifying potential hazards, and health check-up based on work risks, etc. These mechanisms are not found in the informal workers, especially among agricultural migrant workers. Apart from the lack of support for occupational health knowledge, there also be communication problems. Use of language to communicate directly to migrant workers may be a problem and an obstacle, sometimes causes misunderstandings.

Objective The aim of this project is to develop a knowledge mechanism driven by the occupational health leader who can understand both Thai and migrant workers' language in order to provide the right and understandable knowledge by learning materials.

Method The researcher has developed a safety promotion model to have the occupational health leader to create work safety characteristics and behaviours as well as transfer this knowledge to migrant workers. The action research collects samples by mixed methodology, applying both qualitative and quantitative research in order to create improvement. The research uses participation technique along with conducting field experiment to test the effectiveness of tools or equipment or patterns or mechanisms developed with the target group.

Result Occupational health leaders can convey the knowledge of the agricultural chemicals risks, and make the migrant workers aware of potential dangers, know how to use selfprotective equipment from agricultural chemicals and have better health.

\section{ERGONOMIC RISK FACTORS, MUSCULOSKELETAL SYMPTOMS, EXERTION AND WORKLOAD AMONG PHYSIOTHERAPISTS IN A SINGLE REHABILITATION CENTRE}

${ }^{1}$ Joshua Samuel Rajkumar*, ${ }^{2}$ Deepak Sharan. ${ }^{1}$ Department of Orthopaedics, RECOUP Neuromusculoskeletal Rehabilitation Centre, Bangalore, India; ${ }^{2}$ Research and Development, RECOUP Neuromusculoskeletal Rehabilitation Centre, Bangalore, India

\subsection{6/oemed-2018-ICOHabstracts.419}

Introduction Physiotherapists (PTs) apply manual forces such a pushing, pulling and lifting, maintain hazardous postures and static loading during treatment, which predisposes them to work related musculoskeletal disorders (WRMSD). Hence, the aim of this study was to evaluate the risk factors for WRMSDs in a group of PTs working in a rehabilitation centre.

Methods A prospective study was conducted among 120 PTs working in a neuromusculoskeletal rehabilitation centre in an Industrially Developing Country. Musculoskeletal and neurological conditions in adult and paediatric populations were primarily treated in the centre. The PT's were evaluated with a self-reported questionnaire which included demographic data, short-form Work Style Questionnaire, Nordic Musculoskeletal Pain Questionnaire, Borg CR 10 and NASA Task Load Index. The inclusion criteria were: PTs working for a minimum of 6 months of experience in the same centre, treating a minimum 